
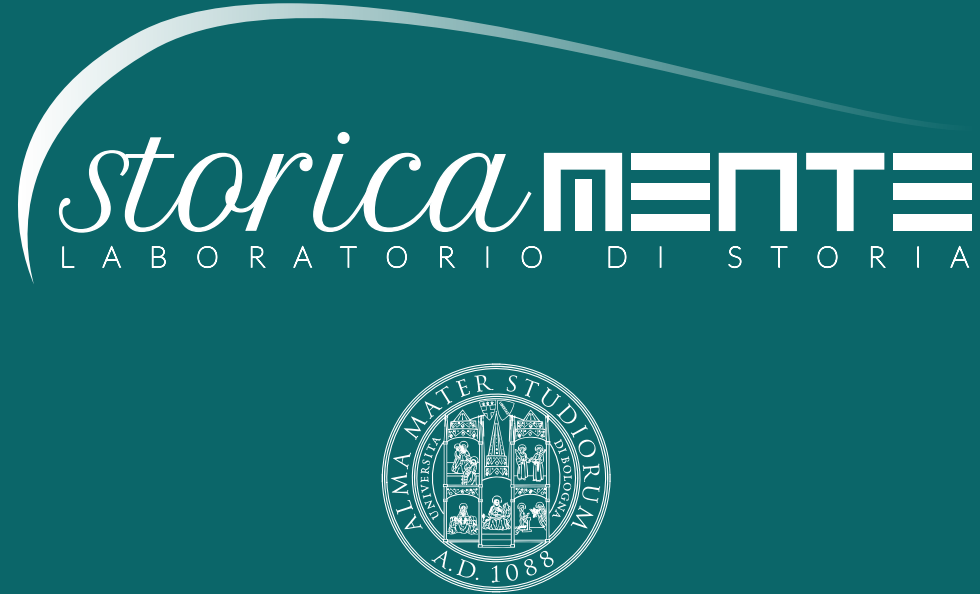

ALMA MATER STUDIORUM

Università di Bologna

Dipartimento di Storia Culture Civiltà

COMUNICARE STORIA

LA STORIA A SCUOLA OGGI.

INSEGNARE STORIA

NELLA SCUOLA PRIMARIA 


\section{STORICAMENTE.ORG}

\section{Laboratorio di Storia}

Elisabetta Serafini

Che genere di Preistoria? Genere e insegnamento della Preistoria nella scuola primaria

Numero $15-2019$

ISSN: $1825-411 \mathrm{X}$

Art. 48

pp. 1-21

DOI: $10.12977 /$ stor794

Editore: BraDypUS

Data di pubblicazione: 25/09/2020

Sezione: Comunicare storia: "La Storia a scuola oggi. Insegnare storia nella scuola primaria" 


\title{
Che genere di Preistoria? Genere e insegnamento della Preistoria nella scuola primaria
}

\author{
ELISABETTA SERAFINI \\ Univ. degli Studi di Roma 'Tor Vergata', \\ Dipartimento di Storia, Patrimonio culturale,
} Formazione e Società

Starting from a reflection on Prehistory teaching in the Italian school, the article explores the possibility of proposing a gender approach since primary school. By bringing girls and boys closer to the sources, it is possible to provide a more varied and complex image of Prehistory with respect to classic textbooks, in order to making it more intelligible and accessible to them, while enhancing differences at the same time. The proposed materials are at times thought of as insights, in other cases they are seen as tools to deconstruct the manual.

\section{La preistoria nelle Indicazioni Nazionali per la scuola primaria}

A partire dalle Indicazioni Nazionali per i Piani di Studio Personalizzati, emanate nel 2004 dalla ministra Letizia Moratti (D.Lgs. n. 59 del 19 febbraio), i contenuti del curricolo di storia assegnati alla scuola primaria e secondaria di primo grado si collocano a scavalco tra $i$ due segmenti del sistema formativo: dalla preistoria alla caduta dell'Impero romano nella scuola primaria, dal medioevo al Novecento nella scuola secondaria di primo grado. In questo modo è stato modificato l'assetto per cui, fino a quel momento, tra scuola primaria e secondaria si percorreva tre volte l'intero arco cronologico. La suddivisione propo- 
sta dalla riforma Moratti è stata mantenuta nei documenti successivi, le Indicazioni per il curricolo del 2007 (D.M. 31/07/2007) e del 2012 (D.M. 16/11/2012). Dunque, stando alla normativa tuttora in vigore, uno dei dieci «Traguardi per lo sviluppo delle competenze al termine della scuola primaria» recita testualmente: «Comprende avvenimenti, fatti e fenomeni delle società e civiltà che hanno caratterizzato la storia dell'umanità dal paleolitico alla fine del mondo antico con possibilità di apertura e di confronto con la contemporaneità» (MIUR 2012, 53). Tuttavia, quanto viene insegnato nella classe terza della scuola primaria si discosta parzialmente dalla pista suggerita dalle Indicazioni, col supporto di libri di testo che propongono contenuti piuttosto differenti da quanto stabilito a livello ministeriale. Generalmente, infatti, il programma di storia si fa iniziare con la nascita dell'Universo e la storia della Terra, per poi passare alla comparsa delle prime forme di vita, soffermarsi sui grandi rettili del Mesozoico, sull'evoluzione delle prime specie di homo e, infine, dedicarsi alla vera e propria preistoria: contenuti che, per la maggior parte, hanno poco a che vedere con la disciplina storica, in quanto rientrano nell'ambito di competenza delle scienze della Terra e di quelle naturali.

Pur accettando di muoversi tra questi argomenti, e tralasciando le riflessioni intorno all'opportunità di tale proposta, è possibile pensare di proporli in chiave di genere? Come farlo? E, soprattutto, perché è importante farlo?

\section{Genere e storia nella scuola primaria}

Le Indicazioni Nazionali (MIUR 2012) non nascono nel segno della prescrittività e, soprattutto per quanto riguarda i contenuti, al corpo docente viene lasciato un ampio margine di libertà nel costruire la programmazione. Stando al quadro educativo ad ampie maglie che esse 
delineano, non saremmo poi così tenute ${ }^{1}$ a considerare la prospettiva di genere nella relazione educativa e nelle specifiche didattiche disciplinari. Nel suddetto documento infatti, come nel precedente del 2007, compaiono sporadici e sommari accenni alla prospettiva di genere e alla sua presa in carico a livello educativo (ivi, 7, 21, 22-23, 32, 53).

Sebbene la questione, agli occhi di chi legifera per la scuola, appaia ancora marginale, è a partire dagli anni Sessanta e Settanta del secolo scorso, in concomitanza con l'affermarsi dei movimenti della seconda ondata femminista, che si è iniziato a gettare luce sugli effetti del sistema educativo nella costruzione dei ruoli di genere. A partire da quella nuova consapevolezza, si imponeva la necessità di dare vita a un nuovo corso per modificare l'iniquità delle relazioni nel percorso formativo e nella società (Gianini Belotti 2018, 136-214). Da allora, se il dibattito intorno a questi temi - pur scemando, talvolta - non si è mai spento, non ha tuttavia prodotto i risultati sperati.

Alcuni provvedimenti legislativi ${ }^{2}$, sconnessi dalle Indicazioni Naziona$l i$, hanno tentato parziali interventi anche in ambito scolastico, ma la questione non è mai stata messa a sistema, né sono stati avviati seri e strutturali piani di formazione e aggiornamento del corpo docente. A ciò si aggiunga la non marginale questione dei libri di testo - sui quali l'attenzione è alta da decenni - che seguitano a presentare lacune importanti, quando non sono ricettacolo di imbarazzanti stereotipi ${ }^{3}$.

Oltre che per ovvie ragioni biografiche, laddove non utilizzo sia il maschile sia il femminile per evitare di appesantire il testo, ho scelto di fare ricorso al solo femminile plurale poiché nel sistema formativo italiano 4 insegnanti su 5 sono donne. Nella scuola primaria la percentuale femminile è oltre il $90 \%$. Si veda il rapporto Ocse Uno sguardo sull'istruzione, al link http://www.oecd.org/education/skills-beyond-school/ EAG2016-Italy.pdf.

Tra gli altri, L. 119/2013, Disposizioni urgenti in materia di sicurezza e per il contrasto della violenza di genere nonché in tema di protezione civile e di commissariamento delle province, art. 5, Piano d'azione straordinario contro la violenza sessuale e di genere, punto c; L. 128/2013, Misure urgenti in materia di istruzione, università e ricerca, art. 16, punto d.

Sui libri di lettura per la scuola primaria si veda l'importante saggio di Biemmi (2009), che racchiude una completa panoramica di quanto accaduto anche negli anni 
La considerazione della categoria di genere nel sistema educativo è questione molto complessa e assume delle specifiche forme se osservata dal punto di vista disciplinare dell'insegnamento della storia. Da questa prospettiva, vanno considerate particolari attenzioni metodologiche, che non siano un fardello quanto un arricchimento in termini di potenzialità per la relazione educativa. Parlare infatti di storia delle donne e di genere a scuola non vuol dire semplicemente aggiungere argomenti al programma, ma cambiare impostazione, per rendere la storia più prossima ai soggetti - a chi l'ha vissuta, a chi contribuisce ogni giorno a ricostruirla con le sue ricerche, a chi la studia a scuola - e per proporla come trait d'union tra essi. Come sostengono le autrici di un manuale francese di storia delle donne "Il s'agit del changer le regard, non de "charger la barque" ". Nello specifico, mutare lo sguardo vuol dire ereditare, prendere in carico la complessità che deriva da questo ambito di studi: rendere visibile la presenza delle donne nel passato ha significato e significa ancora oggi interrogare le fonti in modo differente; ri-valutare ambiti tradizionali della ricerca (storia politica, economica) o considerarne di nuovi (l'intimo, le emozioni, i corpi, le sessualità, l'amore, la maternità), nella consapevolezza che anche il privato può essere oggetto di indagine storica, per gli uomini, per le donne e per gli altri generi; riesaminare le cesure cronologiche; analizzare le relazioni sociali tra donne e uomini, per contestualizzare temporalmente e spazialmente elementi spesso ritenuti frutto dell'organizzazione naturale. Tutto ciò omettendo in questa sede una considerazione a più ampio spettro del genere nella formazione, che necessariamente prevede una serie di altre questioni, come ad esempio la riflessione sulla lingua (Gamberi 2014).

precedenti, e il successivo saggio di Corsini e Scierri (2016); sugli aspetti legislativi e sui sussidiari di storia, Serafini 2019.

"Si tratta di cambiare lo sguardo, non di “caricare la barca” (Dermenjian et al. 2010, 12). 


\section{La preistoria, questa sconosciuta}

Venendo all'ambito specifico di cui in questa sede si intende parlare - la preistoria - sento la necessità di specificare che non si tratta di un mio ambito diretto di ricerca. Piuttosto, me ne occupo da qualche anno non solo per aggiornare le mie sempre lacunose conoscenze (e competenze) di docente di scuola primaria, vista la vastità di discipline e di contesti coi quali ci si deve misurare in queste vesti, ma anche in virtù di un progetto che ho la fortuna di coordinare e che, dal 2015, vede collaborare la Società italiana delle storiche ${ }^{5}$ e la casa editrice Settenove ${ }^{6}$ per la realizzazione della collana di albi illustrati Storie nella storia?.

Dedicarsi all'approfondimento della preistoria in chiave di genere implica il doversi spingere necessariamente oltre i confini nazionali, poiché, se per gli studi di storia delle donne e di genere relativi alle età medievale, moderna e contemporanea la storiografia italiana è nutrita

La Società italiana delle storiche è stata fondata nel 1989 con l'obiettivo di promuovere la ricerca storica, didattica e documentaria nell'ambito della storia delle donne e della storia di genere (http://www.societadellestoriche.it/index.php?option=com_con tent\&view=featured\&Itemid=174, ultimo accesso 25/08/2020).

${ }^{6} \quad$ La casa editrice è nata nel 2013 come "primo progetto editoriale italiano interamente dedicato alla prevenzione della discriminazione e della violenza di genere». Il nome che è stato scelto per il progetto fa riferimento diretto all'anno 1979, «durante il quale le Nazioni Unite hanno adottato la Cedaw, la Convenzione Onu per l'eliminazione di ogni forma di discriminazione e violenza contro le donne, che per la prima volta individua nello stereotipo di genere il seme della violenza» (http://www. settenove.it/chi-siamo, ultimo accesso 25/08/2020).

La collana è nata dalla volontà di raccontare la storia delle donne e di genere alle giovani generazioni, ponendola in relazione alla storia tradizionalmente studiata a scuola. I volumi che compongono la collana coprono l'intero arco temporale che va dalla preistoria alla contemporaneità, si rivolgono a giovani lettrici e lettori tra gli 8 e i 13 anni, e sono caratterizzati da complessità crescente. Sono previsti nel progetto anche approfondimenti su temi cruciali per la storia delle donne e delle relazioni di genere (come il lavoro, la cittadinanza, la famiglia) in volumi tematici. Questa scansione è data dalla volontà di seguire quella definita dalle Indicazioni Nazionali per consentire un uso didattico dei volumi. Quanto è scritto in queste pagine è frutto delle ricerche compiute per la progettazione del volume sulla preistoria, a cura della sottoscritta (Serafini 2018). 
e affermata nel panorama internazionale, lo stesso non può dirsi per la preistoria e la storia antica, fatti salvi sparuti studi e, ovviamente, la storia romana. Contemporaneamente, comporta la considerazione di un immaginario comune costruitosi su una limitata conoscenza di quella storia così lontana e così poco (e male) studiata nella scuola italiana. In effetti nel sistema scolastico precedente alla riforma Moratti la preistoria sarebbe dovuta comparire nei programmi della scuola elementare, media inferiore e superiore; ma, di fatto, si studiava soltanto nella scuola elementare. Con l'attuale assetto si dovrebbe tornare su di essa nel primo anno della scuola secondaria di secondo grado, dopo averla affrontata alla primaria. Anche in questo caso, nella scuola secondaria ci si sofferma assai poco su di essa per dedicarsi maggiormente alla storia antica, come si può riscontrare negli indici dei manuali (Tarantini 2008, 76-77). Inoltre, nella scuola primaria - come si è visto - lo spazio che potrebbe essere dedicato a temi propriamente preistorici, dal Paleolitico al Neolitico, viene mangiato quasi completamente dalla geologia e da argomenti che si ritengono più accattivanti per bambine e bambini (orientati in questa direzione anche dal mercato), come lo studio dei dinosauri, che, si è detto, poco hanno a che fare con la storia. Eppure si tratta di un capitolo della storia dell'umanità importante non solo per i suoi ampi margini temporali, ma perché in quel lungo periodo hanno avuto luogo trasformazioni determinanti attraverso le quali ha preso forma il mondo per come ancora oggi lo conosciamo. Penso in particolare alla rivoluzione agricola del Neolitico.

Oltre alla scarsa presenza della preistoria nel sistema formativo, il suo insegnamento - si può dire quasi esclusivo - nella scuola primaria è molto spesso supportato da libri di testo scritti da non specialisti, nei quali emergono ricorrenti stereotipi su questo lunghissimo periodo della storia dell'umanità. Stereotipi che, spesso e volentieri, rimangono le nostre poche conoscenze sulla preistoria anche in età adulta. 
Quanto finora detto non può, ovviamente, non tenere in considerazione la scarsa preparazione, inziale e in servizio, del corpo docente su questi temi, che si tratti di scuola primaria o secondaria.

Eppure, nonostante il panorama desolante, la ricerca in ambito preistorico continua a fornire interessanti e nuove prospettive, che faticano a trovare spazio nelle aule. Questa abbondanza e varietà è propria anche degli studi che intendono ricostruire il mondo preistorico come un mondo popolato di uomini e donne senza calare su di esso schemi e filtri propri della contemporaneità. Nelle righe che mi restano proverò a offrire una prospettiva di innesto tra questi studi e la pratica didattica.

\section{Insegnare la preistoria in chiave di genere}

L'ambito preistorico, per alcuni aspetti, si presta molto bene a una ricostruzione "equa" del passato (intendo equa come rappresentativa della società nel suo complesso), dal punto di vista del genere, poiché le fonti, materiali, che restano sono state indistintamente lasciate da donne e uomini, a differenza di quelle scritte - essendo spesso la scrittura appannaggio dei soli uomini -, anche a fronte della loro scarsa disponibilità. Eppure, fino a un dato momento, quando le interpretazioni delle fonti venivano prodotte in un mondo il cui immaginario riconduceva solo al maschile l'ambito del fare, dell'agire, la preistoria appariva come un mondo popolato di soli uomini.

Oggi, un cambiamento di prospettiva, unitamente al progredire del sapere e a un approccio interdisciplinare, permette di restituire una lettura diversa di quegli eventi così distanti. Un esempio paradigmatico di reinterpretazione di fonti già note alla luce di nuove conoscenze e strumentazioni riguarda le pitture rupestri del Paleolitico superiore.

Qualche anno fa l'archeologo Dean Snow, della Pennsylvania State University, facendo riferimento agli studi del biologo britannico John Manning, secondo i quali le mani di donne e uomini adulti differiscono 
tra di loro per la lunghezza delle dita, ha provveduto ad analizzare le impronte di mani tipiche delle pitture rupestri dell'Europa sud-occidentale (precisamente nord della Spagna e sud della Francia). Queste impronte non sono state lasciate casualmente ma sono state ottenute poggiando la mano alla parete e soffiandoci sopra dei pigmenti, così da avere un negativo della loro sagoma: una sorta di stencil preistorico, forse una firma apposta accanto a pitture rappresentanti animali

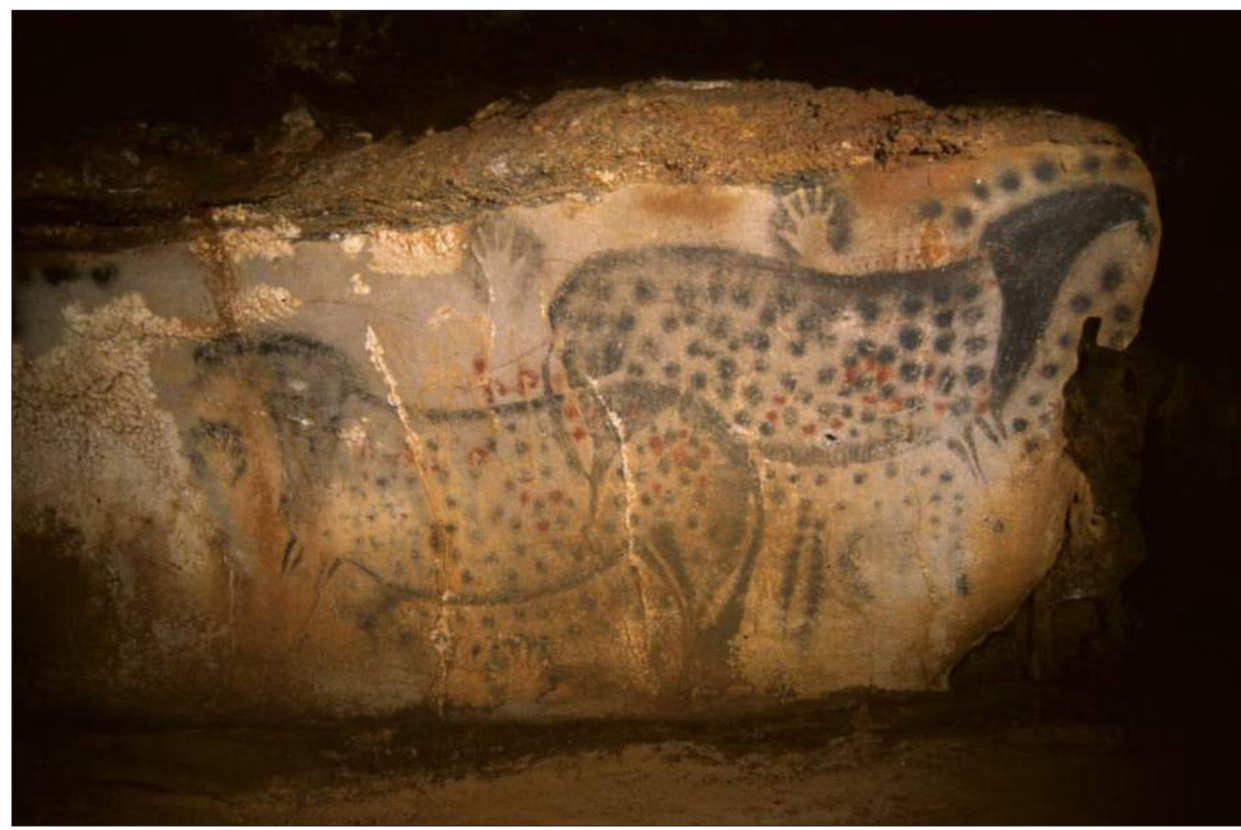

Pitture rupestri della grotta di Pech Merle nei Pirenei francesi, 25.000 anni fa ca. Provenienza: https://archaeology-travel.com/france/pech-merle-cave/

e, meno frequentemente, scene di caccia. Interpretazioni precedenti a quella di Snow davano per scontato che a realizzarle fossero stati gli uomini cacciatori, autori delle pitture di cui erano a margine e raffiguranti mammut, bisonti e altri animali. L'archeologo della Pennsylvania University ha invece potuto calcolare con un soddisfacente margine di accuratezza che quelle impronte appartengono a donne in due terzi dei casi (Di Barbora 2019). Il dato di per sé non fornisce alcuna certezza, 
ma è possibile ipotizzare che a eseguire le pitture rupestri spesso fossero le donne, poiché, pur essendo la caccia ai grandi animali condotta prevalentemente da uomini, anch'esse la conoscevano molto bene, visto che si occupavano della macellazione e della sistemazione delle carni. Questa prospettiva ci offre un quadro maggiormente diversificato rispetto a quello che ci viene fornito generalmente dai libri di testo per la scuola primaria, nei quali le attività vengono suddivise in modo più netto in base al genere: la caccia attribuita agli uomini, la raccolta alle donne. Quello che spesso viene proposto come un assioma è ancora meno valido in società dedite prevalentemente alla raccolta, per le quali gli studi hanno dimostrato una minore polarizzazione e una certa intercambiabilità nello svolgimento delle attività (Ehrenberg 1992, 57-61). Oltre alla reinterpretazione di fonti già note, disponiamo di nuove scoperte che contribuiscono a ricomporre un passato talmente lontano temporalmente e culturalmente, da indurre talvolta ad applicare paradigmi del presente a ciò che più mette a dura prova la nostra capacità di comprensione. Prima di passare a una breve presentazione di qualche ritrovamento particolarmente interessante per la prospettiva qui assunta, si può fare un esempio per comprendere meglio questa "trappola culturale" sempre in agguato. Sono note in tutto il mondo le impronte di Laeotoli, scoperte nel 1978 dalla paleantropologa Mary Leakey nell'area protetta di Ngorongoro, in Tanzania, e risalenti a 3,6 milioni di anni fa. Si tratta delle impronte lasciate su uno strato di ceneri vulcaniche umide appartenenti a tre individui di Australopithecus afarensis (la stessa specie della famosa Lucy), di taglie diverse tra loro che avanzavano con postura bipede. Trattandosi di tre individui di cui uno di taglia più grande e due più piccoli, si ipotizzò che, in base alla teoria del dimorfismo sessuale ${ }^{8}$, si trattasse di un maschio seguito da una femmina e

Il dimorfismo sessuale è un fenomeno per il quale individui appartenenti ai due sessi della stessa specie presentano caratteristiche fisiche diverse (ad esempio, le maggiori dimensioni del maschio rispetto alla femmina). 
un cucciolo, che camminavano a stretto contatto: un rassicurante quadro familiare ricostruito nella preistoria. Una scoperta recente, emersa da studi condotti dalla scuola di Paleoantropologia dell'Università di Perugia, in collaborazione con ricercatori delle Università "Sapienza" di Roma, Firenze, Pisa e Dar es Salaam, ha permesso di individuare, in prossimità delle impronte scoperte negli anni Settanta, quelle di altri due individui, di cui uno incredibilmente più grande degli altri. Si tratta dell'Australopithecus più grande sinora ritrovato, con una statura stimata di 1.65 metri. Molto probabilmente si trattava di un unico gruppo di ominidi in movimento nella savana composto da un maschio, due/ tre femmine e uno/due giovani: un'organizzazione sociale e delle strategie riproduttive più simili a quelle degli attuali gorilla, scimmia ad alto dimorfismo sessuale e poligama, piuttosto che a specie moderatamente dimorfiche promiscue o monogame. Ciò a dimostrare che l'applicazione del modello "tradizionale" di "famiglia" (termine che usiamo tra l'altro erroneamente in luogo di "gruppo"), per come si presenta nel nostro immaginario, perché - di fatto - non possiamo ascriverlo in modo univoco neanche al nostro presente, non è applicabile a tutte le coordinate spazio-temporali (Masao et al. 2016).

Tornando al discorso relativo alla minore polarizzazione delle attività e alle nuove scoperte, ci vengono di nuovo in aiuto fonti attraverso le quali viene messo in discussione il paradigma dell'uomo cacciatore. Prima di procedere è però bene ricorrere ad alcune specificazioni.

Fino a che le specie di homo si trovarono ad abitare le zone temperate, e cioè prevalentemente l'Africa, la loro sussistenza era legata più alla raccolta che alla caccia. La prima infatti assicurava un maggior numero di calorie a fronte di minori rischi. I gruppi erano dunque prevalentemente gruppi di raccoglitori che si dedicavano occasionalmente alla caccia, prevalentemente di piccoli animali, e lo facevano anche in gruppo. Questo equilibrio vide un primo cambiamento con l'uscita dall'Africa e il popolamento di continenti più freddi, dove si riuscivano a reperire vegetali in minor quantità e vivevano grandi mammiferi. 


\section{L'infanzia nella preistoria}

Quelle zone temperate dell'Africa orientale, e più precisamente il sito di Melka Kunture, in Etiopia, nei primi mesi del 2018 sono state lo scenario di un ritrovamento eccezionale, compiuto da un'équipe di studiosi dell'Università "Sapienza" di Roma. Si tratta di impronte che hanno consentito di ricostruire molte informazioni su una battuta di caccia all'ippopotamo compiuta da un gruppo appartenente alla specie Homo heidelbergensis (antenata comune di Neanderthal e Sapiens) circa 700.000 anni fa. Dopo due anni di campagne di ricerca è stato possibile individuare un'area un tempo occupata da una pozza d'acqua, per questo frequentata non solo da ominidi ma anche da animali di varie specie, tra cui piccoli uccelli, gazzelle e ippopotami. È possibile affermare ciò in virtù delle tracce lasciate su un terreno fangoso e poi fossilizzatesi

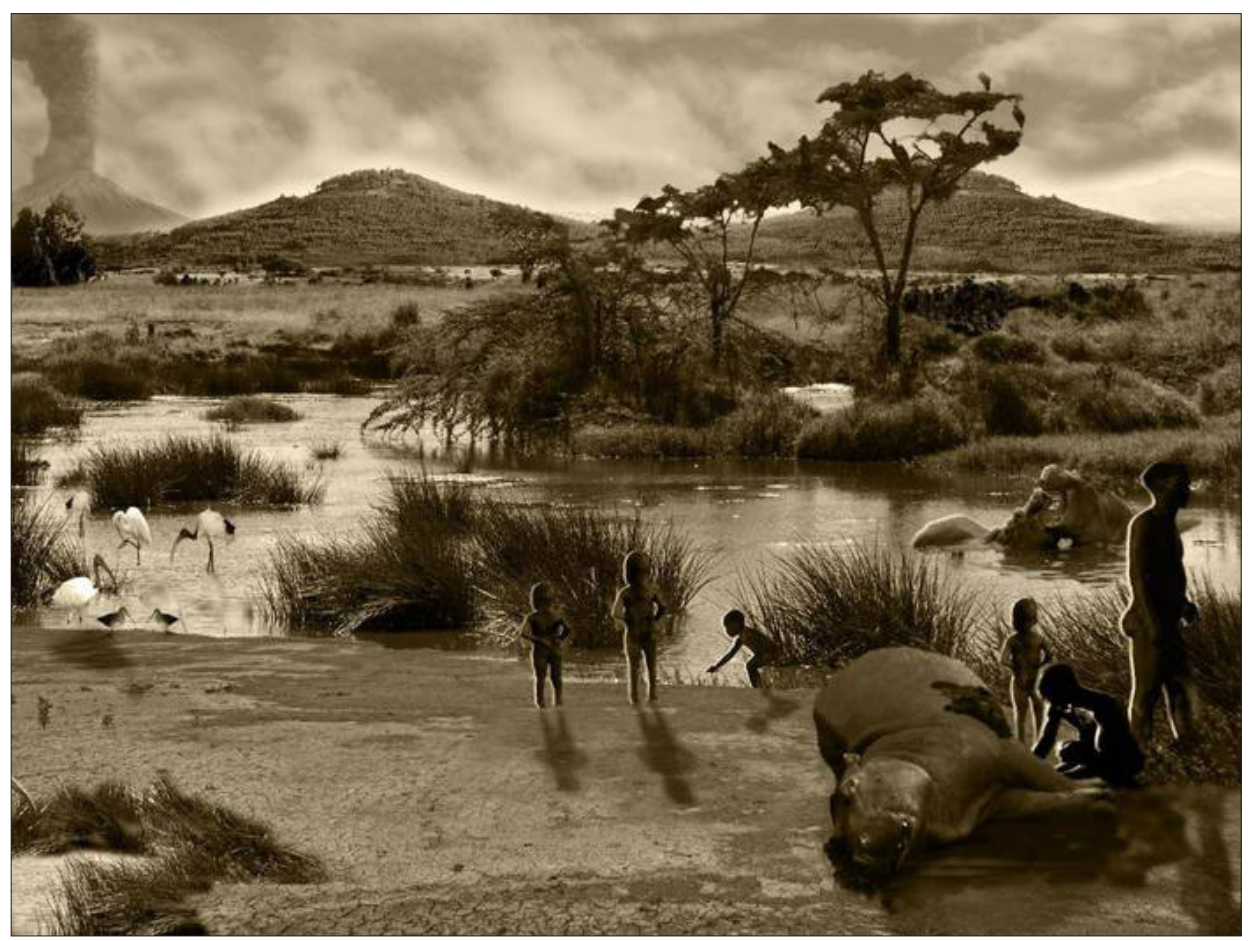

Ricostruzione del sito di Melka Kunture (Matthew Bennett), 2018. Provenienza: https://www. ansa.it/canale_scienza_tecnica/notizie/terra_poli/2018/02/16/orme-di-baby-cacciatori-rivelanolinfanzia-nella-preistoria-_9bfc13aa-4a7e-40c4-aa1c-5e1306601a6d.html 
e conservatesi fino ai nostri giorni perché protette dalle ceneri prodotte da un'eruzione vulcanica: circostanze che si verificano in rarissimi casi, considerato il limitato numero di siti archeologici che conserva impronte così antiche. L'eccezionalità del ritrovamento consiste anche nel fatto che non si tratta di impronte che rivelano un passaggio di ominidi, quanto piuttosto fermano nel tempo un'attività della vita quotidiana in tutte le fasi che la compongono. Nel sito sono presenti infatti tracce di varie azioni legate alla caccia e alla macellazione di ippopotami, come la scheggiatura di pietre per la realizzazione degli utensili necessari alla macellazione delle carni di più esemplari di essi. Gli strumenti litici hanno lasciato traccia sulle carcasse dei grandi mammiferi fluviali, ma non sono stati i soli. Infatti, dopo gli ominidi, altri carnivori si sono avventati sulle prede per completare l'opera, mangiando le carni residuali. Le impronte di ominidi non rivelano la sola presenza di adulti, ma anche quella di bambini di 1, 2 e 3 anni. Tra questi, uno dei più piccoli non si reggeva ancora bene in equilibrio, come dimostrano le sue impronte incerte sul terreno. Anche se non è possibile stabilire il suo sesso, l'équipe ha voluto vedere in quelle impronte una bambina e chiamarla "Abeba" (che in lingua etiope amarica vuol dire "fiore").

Fino a poco fa si trattava delle più antiche impronte di bambini così piccoli e ciò dimostra che la caccia poteva essere un'attività di gruppo alla quale partecipavano anche i componenti in tenera età, i quali - attraverso l'osservazione prima, la pratica in seguito - apprendevano le tecniche necessarie alla sopravvivenza (Altamura et al. 2018).

Recente è la notizia che, nell'ambito degli stessi scavi, sono state rinvenute nuove impronte infantili ai margini di un fiume che oggi non c'è più. Tufi vulcanici hanno fatto sì che si potessero conservare fino a oggi impronte risalenti a un periodo compreso tra 1,2 milioni e 850.000 anni fa. Sugli strati riportati alla luce sono presenti impronte lasciate da iene, alcuni erbivori simili agli attuali gnu, gazzelle, ippopotami, piccoli molluschi e anche impronte umane, molte delle quali riconducibili a bambini e adolescenti delle specie Homo erectus/ergaster o, forse, 
Homo heidelbergensis arcaico. Con buona probabilità, bambine e bambini preistorici entravano in acqua per esigenze pratiche - bere, lavarsi, catturare pesci con il solo ausilio delle mani - ma anche, come avviene oggi, per giocare (Altamura et al. 2020).

Questi studi ci forniscono l'opportunità non solo di parlare di bambini e bambine nella preistoria, avvicinando i contenuti della disciplina ai soggetti ai quali li proponiamo, ma anche di riflettere insieme a loro sul diverso modo di declinare l'infanzia e la pericolosità a seconda del contesto geografico o del momento storico. Attività simili, che costituivano la vita quotidiana dell'infanzia nel Paleolitico, sarebbero impensabili per bambine e bambini di oggi. A voler dare un taglio di genere, un'ulteriore riflessione potrebbe essere condotta sulla pericolosità in relazione al sesso: le insidie per la sicurezza di un bambino o di una bambina sono in uguale misura? Sono della stessa natura? Perché?

Purtroppo studi come questi, oltre a essere pochi, sono pressoché sconosciuti. Ma è possibile fare qualche altro esempio utile a parlare di infanzia nella preistoria.

Un gruppo di esperti si è dedicato allo studio delle impronte digitali rinvenute su alcune piccole statue raffiguranti animali trovate in Arizona. L'analisi accurata delle impronte ha consentito di stabilire che a plasmare quelle statuine siano stati bambini e bambine, probabilmente coloro che li hanno successivamente utilizzati come giocattoli (Kamp et al. 1999).

Manufatti risalenti al periodo tra il Neolitico e l'età del Bronzo, provenienti dalla Baviera, raccontano invece in che modo venissero alimentati bambini e bambine in quel periodo, individuando in questo caso un elemento di continuità con il presente. Si tratta di piccoli vasi in ceramica a forma di animali, reali o immaginari, che presentano un beccuccio. L'esame delle incrostazioni presenti al loro interno ha permesso di stabilire che un tempo il loro contenuto fosse latte di ruminante o latte umano. Considerando questo elemento, insieme alla forma dei vasi - sono tutti facilmente afferrabili da piccole mani e talvolta sono 
dotati di un doppio manico agli estremi opposti - e al fatto che sono stati rinvenuti presso tombe infantili, fa ipotizzare che fossero una sorta di "biberon" preistorici, utilizzati probabilmente per lo svezzamento o in alternativa al latte materno. Non è un caso che questo filo rosso - che congiunge idealmente il presente con un passato molto lontano - possa essere tracciato a partire dal momento in cui la trasformazione agricola era in atto, e la società cominciava ad assomigliare a quella che sarebbe stata, mutatis mutandis, fino alla rivoluzione industriale e oltre.

Anche se a partire da fonti di diverse epoche e provenienti da contesti differenti, è possibile ragionare insieme alla classe sui mutamenti e sulle persistenze del divenire storico, individuando uno dei nuclei fondanti della disciplina. Alcune delle suddette fonti possono essere messe al centro di una riflessione comune, ancor prima di fornire una spiegazione dettagliata che le riguardi da parte delle insegnanti, che induca la classe a fare delle ipotesi sulla loro funzione nelle società di provenienza. (Dunne et al. 2019).

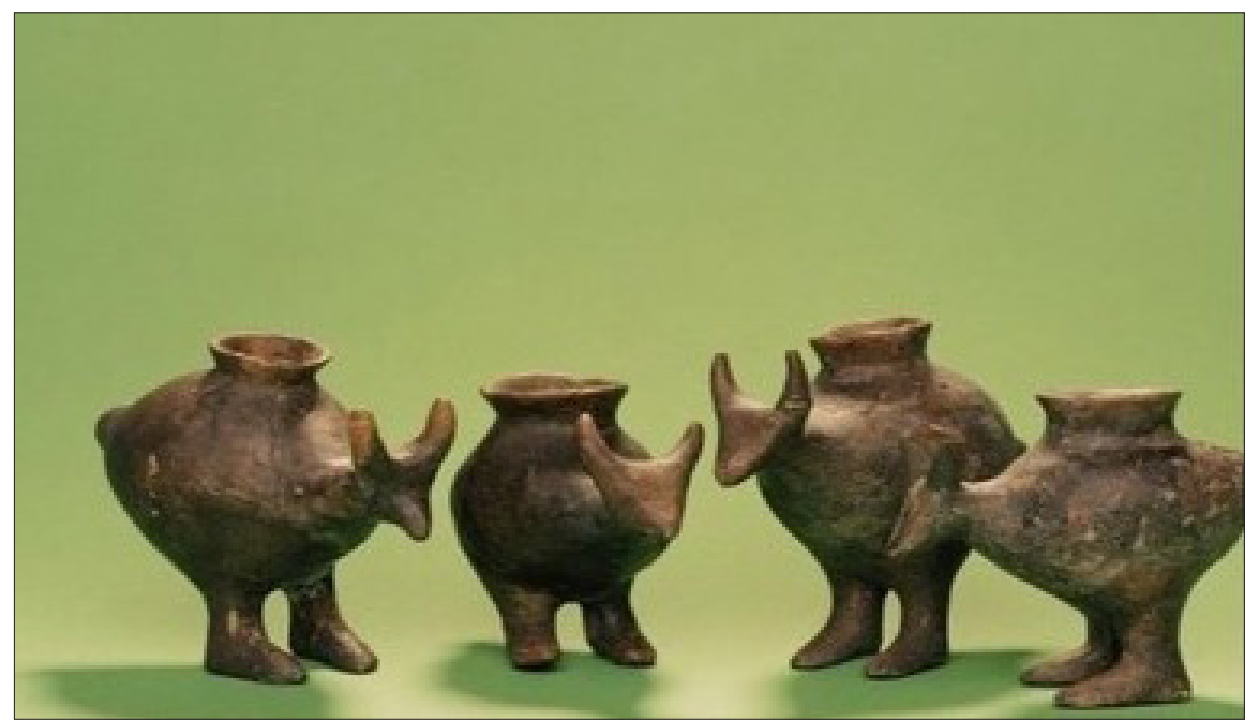

Vasetti e biberon rinvenuti in sepolture dell'Età del Bronzo, nel sito archeologico di Vösendorf (Austria), 1200-450 a.C. ca. Provenienza: https://www.focus.it/cultura/storia/biberon-preistoriciallattamento-svezzamento-dei-bambini-nel-neolitico 


\section{Donne nella preistoria: l'immaginario e la realtà}

Se talvolta le tecnologie consentono di acquisire informazioni chiave per interpretare in modo convincente le fonti, in altri casi interpretazioni apparentemente persuasive rivelano la loro fragilità nel momento in cui si assume una prospettiva differente. Una tipologia di fonte utile a illustrare questo passaggio è rappresentata dall'arte rupestre. Esempi rinvenuti in Scandinavia e sulle Alpi meridionali risalgono all'età del Bronzo, e riproducono scene in cui esseri umani sono dediti a varie attività. Le figure sono delineate in modo rozzo (va considerata la difficoltà a incidere superfici di granito), per cui non è facile distinguere gli uomini dalle donne. Alcune di quelle persone sono rappresentate coi capelli lunghi e questo, in un primo momento, ci indurrebbe a considerarle di sesso femminile, facendo riferimento alle abitudini e ai canoni estetici della nostra epoca, ma non possiamo darlo per scontato (Erhenberg 1992, 221-6). Lo stesso si può dire per l'interpretazione delle incisioni su ceramiche provenienti da Sopron, in Ungheria, e risalenti con buona probabilità al VI sec. a.C. Si tratta di vasi, probabilmente urne cinerarie, sulle quali sono rappresentate donne intente in varie attività. Donne perché sembrano indossare ampie gonne ma non si può avere la certezza che quelle che, ai nostri occhi, sembrano gonne, non siano invece ampi mantelli (ivi, 236-9).

Un'attività interessante e rivelatrice potrebbe essere condotta con la classe proponendo di interpretare le suddette scene, per tentare di individuare il sesso delle persone rappresentate e che tipo di attività stessero svolgendo; un buon metodo è quello di lasciar esprimere bambine e bambini senza intervenire, registrando le loro riflessioni, e poi provare a suggerire letture differenti, facendo notare che, anche oggi, abbigliamento e acconciature possono assumere forme diverse nei vari contesti nazionali e regionali. L'interesse di questa proposta risiede nella possibilità di essere una cartina tornasole delle griglie interpretative che vengono utilizzate da bambini e bambine per imparare insieme a riconoscerle, discuterne e decostruirle. 
Avvicinarsi alle fonti consente inoltre di intravedere una realtà molto più complessa rispetto a quella polarizzata che viene proposta dai libri di testo. Questi spesso confinano la presenza femminile ad alcuni ambiti, attribuendo ad esempio alle donne un ruolo fondamentale nella "scoperta” dell'agricoltura senza aver riconosciuto loro un apporto determinante nella raccolta oppure, andando a ritroso, raccontando la centralità della figura femminile nelle società preistoriche attraverso le cosiddette "Veneri".

Le statuette sono spesso state studiate come «fenomeno unico», cioè univocamente rappresentanti divinità femminili che starebbero a dimostrare una centralità delle donne nelle società. È però im-

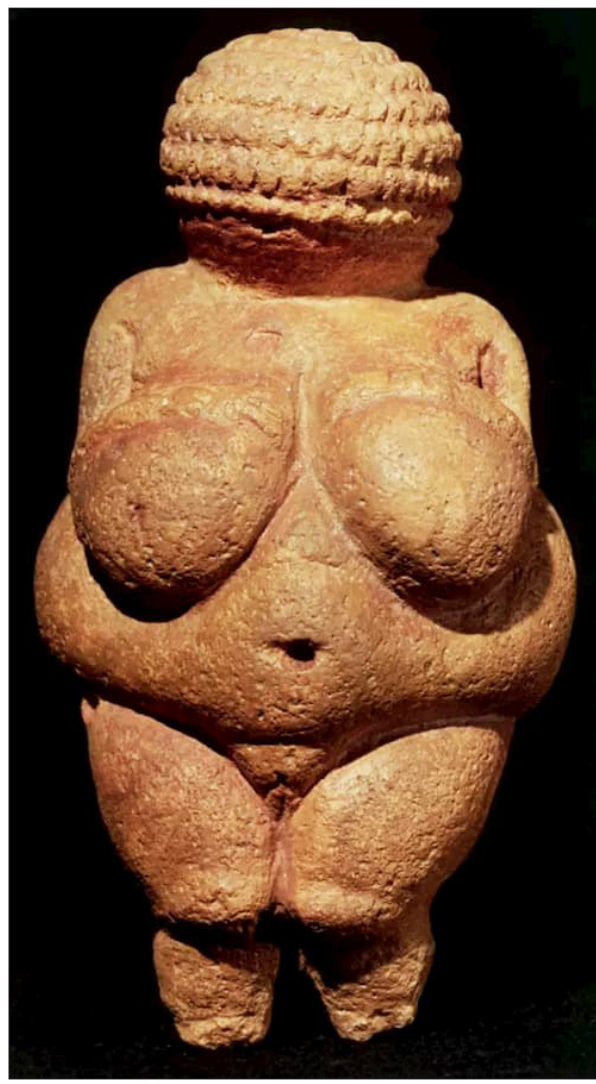

Venere di Willendorf, 23.000-19.000 a.C., Naturhistorisches Museum (Vienna), Provenienza: https://www.arteworldit/venere-di-willendorfanalisi/ portante precisare innanzitutto che la loro produzione si distribuisce su un periodo di tempo molto ampio - dal Paleolitico superiore (circa 25.000 a.C.) all'età del Bronzo (circa 2000 a.C.) - e su diverse aree geografiche, anche molto distanti tra loro; e aggiungere che esse fanno parte di una produzione che comprende statuette rappresentanti esseri umani di sesso maschile o di sesso non chiaramente identificabile, di animali o anche di strane creature non riconducibili a esseri viventi. Si può escludere che statuine così eterogeneamente distribuite possano indicare la presenza di un diffuso culto della dea madre e, di conseguenza, segnalare una posizione cen- 
trale delle donne all'interno delle comunità sembra abbastanza improbabile. Va considerato poi anche il fatto che, in molti casi, sono assenti altre prove normalmente associate a tale culto, ma soprattutto che il loro ritrovamento è avvenuto in contesti molto diversi tra loro: templi, sepolture ma anche abitazioni e cumuli di rifuti. Infine, non si può essere certi, senza ulteriori conferme archeologiche, che la presenza di un culto femminile indichi una posizione preminente delle donne nella società.

Nell'Europa paleolitica è quindi difficile immaginare un culto generalizzato basato su una specifica divinità femminile, sia per gli sporadici contatti tra le comunità, sia per la struttura di queste ultime: anche ai nostri giorni la spiritualità dei popoli raccoglitori è basata su divinità generiche. Esse potrebbero raffigurare donne che noi riteniamo obese ma che ai tempi erano ritenute attraenti e aventi un nesso con la fertilità (importante per la sopravvivenza della specie). Potrebbero aver rappresentato spiriti deputati alla protezione della casa e del focolare (laddove sono state trovate nelle abitazioni); nelle rappresentazioni più recenti, persone devote o sacerdotesse; personaggi pseudo-storici (in alcune zone dell'Africa ancora oggi si usano statue simili a scopo didattico, per illustrare riti di iniziazione, personaggi del mito o comportamenti corretti da tenersi in comunità).

L'ipotesi più probabile è che questi oggetti fossero realizzati per propiziare la fertilità, supposizione attestata da molte ricorrenze etnografiche e corroborata dal fatto che in quelle società non era chiaro il nesso tra fecondazione e nascita. Inoltre, sia nel Paleolitico sia nel Neolitico, le statue sono state ritrovate tra i rifiuti: il fatto avvalora l'ipotesi che non si trattasse di divinità e che, una volta svolta la loro funzione (la gravidanza), il loro ruolo fosse esaurito (ivi, 100-17).

La considerazione di questi elementi renderà possibile arricchire, ma anche contraddire in parte, i contenuti dei libri di testo. Troppo spesso demonizzati, essi restano - a mio avviso - uno strumento fondamentale per le formazione. Sono anzitutto il primo contatto che bambine 
e bambini hanno con il "manuale", un compendio del sapere pensato a scopo didattico presente a ogni livello dell'apprendimento. Non possiamo però, come insegnanti, perdere l'occasione di esplorarlo con la classe per conoscerne potenzialità e limiti intrinseci: proporne un uso consapevole fin dalla scuola primaria vuol dire stimolare la coscienza critica necessaria a servirsene come strumento da un lato da esplorare e analizzare per potersi orientare nella storia, dall'altro da decostruire e arricchire.

\section{L'uso delle biografie}

L'insegnamento della preistoria in chiave di genere, facendo riferimento a una ricostruzione della vita quotidiana attraverso tracce lasciate molto spesso involontariamente, allontana dal rischio di presentare la storia delle donne come mera rassegna di vite illustri: «l'archeologia ci racconta la preistoria di tutte le donne e di tutti gli uomini» (ivi, 15). Le biografie di donne eccezionali per la loro epoca sono sì importanti, anche perché possono fungere da modelli di riferimento, ma rischiano di passare come esempi di situazioni straordinarie rispetto a una "normalità" messa ancor più in ombra dall'emergere di isolati casi particolari. Credo tuttavia che presentarne alcune emblematiche, anche in questo contesto, possa essere utile a far riferimento agli studi sul passato e a chi li ha condotti, aspetto totalmente espunto dai programmi per la scuola primaria, oltre che ad alcune specifiche questioni di genere. Richiamo, a titolo di esempio, Mary Anning e Mary Leakey. Attraverso la prima, autodidatta "cercatrice" di fossili di dinosauri (1799-1847), si può affrontare, in una prospettiva differente, la presentazione dei grandi rettili del Mesozoico (Strada e Tieni 2019). Con le ricerche di Mary Leakey l'attenzione si volge invece all'individuazione dell'Africa come zona di provenienza degli antenati della nostra specie, avvenuta nel XX secolo, a differenza di quanto si riteneva precedentemente (Pulcinelli e Fac- 
chini 2008). La ricezione degli studi di queste "cercatrici di fossili" e la loro diffusione al di fuori dell'ambito specialistico può essere elemento di riflessione sull'esclusione delle donne dai contesti ufficiali del sapere fino al XX secolo, e sul rapporto con le "scienze dure" che ancora oggi ne determina scelte e carriera.

Se, a partire dalla riforma Moratti, l'insegnamento sistematico della storia medievale, moderna e contemporanea è stato estromesso dalla scuola primaria, bambini e bambine sottopongono continuamente questioni per rispondere alle quali le insegnanti non possono fare a meno di attraversare anche quegli ambiti. Rispetto alle domande che circolano nelle classi, la preistoria appare spesso come un passato molto lontano e, per questo, difficile da raccontare. Forse l'approccio di genere può essere uno strumento utile a rendere la materia più accessibile, ma anche più accattivante, e l'apprendimento più significativo, oltre che provare a riallineare ciò che viene insegnato a scuola con i progressi della ricerca.

\section{Bibliografia}

Altamura, Flavio, Matthew R. Bennett, Kristiaan D'Août, Sabine Gaudzinski-Windheuser, Rita T. Melis, Sally C. Reynolds, e Margherita Mussi. 2018. "Archaeology and ichnology at Gombore II-2, Melka Kunture, Ethiopia: everyday life of a mixed-age hominin group 700,000 years." Scientific Reports 8 (2815) (DOI:10.1038/ s41598-018-21158-7).

Altamura, Flavio, Matthew R. Bennett, Lorenzo Marchetti, Rita T. Melis, Sally C. Reynolds, e Margherita Mussi. 2020. "Ichnological and archaeological evidence from Gombore II OAM, Melka Kunture, Ethiopia: an integrated approach to reconstruct local environments and biological presences between 1.2-0.85 Ma.” Quaternary Science Reviews 244 (106506) (https://doi.org/10.1016/j.quascirev.2020.106506).

Biemmi, Irene. 2010. Educazione sessista, Stereotipi di genere nei libri delle elementari. Torino: Rosenberg \& Sellier.

Corsini, Critiano, e Irene D.M. Scierri. 2016. Differenze di genere nell'editoria scolastica: indagine empirica sui sussidiari dei linguaggi per la scuola primaria. Roma: Nuova Cultura. 
Dermenjian, Geneviève, Irène Jami, Annie Rouquier, e Françoise Thébaud, a cura di. 2010. La place des femmes dans l'histoire. Une histoire mixte. Paris: Belin.

Di Barbora, Monica. 2019. "Fonti iconografiche." In I secoli delle donne. Fonti e materiali per la didattica della storia, a cura di Franca Bellucci, Alessandra F. Celi e Liviana Gazzetta, 243-54. Roma: Biblink.

Dunne, Julie, Katharina Rebay-Salisbury, Roderick B. Salisbury, Alexander Frisch, Caitlin Walton-Doyle, e Richard P. Evershed. 2019. "Milk of ruminants in ceramic baby bottles from prehistoric child graves.” Nature 574: 246-8 (https://doi. org/10.1038/s41586-019-1572-x).

Ehrenberg, Margaret. 1992. La donna nella preistoria. Milano: Mondadori.

Gamberi, Cristina. 2014. "Ripensare la relazione educativa in un'ottica di genere. Riflessione teorica e strumenti operativi." In La differenza insegna. La didattica delle discipline in una prospettiva di genere, a cura di Maria Serena Sapegno, 13-22. Roma: Carocci.

Gianini Belotti, Elena. 2018. Dalla parte delle bambine. Milano: Feltrinelli, ed. digitale.

Kamp, Kathryn A., Nichole Timmerman, Gregg Lind, Jules Graybill, e Ian Natowsky. 1999. "Discovering childhood: using fingerprints to find children in the archaeological record." American Antiquity 64 (2): 309-15 (https://doi.org/10.2307/2694281).

MIUR. 2012. "Indicazioni Nazionali per il Curricolo della Scuola dell'Infanzia e del Primo Ciclo d'Istruzione". Annali della Pubblica Istruzione LXXXVIII (Numero Speciale). (http://www.indicazioninazionali.it/wp-content/uploads/2018/08/Indicazioni_Annali_Definitivo.pdf)

Indicazioni Nazionali per il Curricolo della Scuola dell'Infanzia e del Primo Ciclo d'Istruzione. 2012. (Annali della Pubblica Istruzione n.s.). Firenze: Le Monnier.

Masao, Fidelis T., Elgidius B. Ichumbaki, Marco Cherin, Angelo Barili, Giovanni Boschian, Dawid A. Iurino, Sofia Menconero, Jacopo Moggi-Cecchi, e Giorgio Manzi. 2016. "New footprints from Laetoli (Tanzania) provide evidence for marked body size variation in early hominins." eLife (https://doi.org/10.7554/eLife.19568.001).

Pulcinelli, Cristiana e Vittoria Facchini. 2008. Alla ricerca del primo uomo: storia e storie di Mary Leakey. Trieste: Editoriale Scienza.

Serafini, Elisabetta. 2018. Preistoria. Altri sguardi, nuovi racconti. Cagli: Settenove.

Serafini, Elisabetta. 2019. "Che genere di storia? Indagine sui manuali della scuola primaria e secondaria di primo grado.” In Tra storia e storie, a cura di Anna Antoniazzi e Maria Lucenti, 65-86. Reggio Emilia: Junior.

Strada, Annalisa, e Daniela Tieni. 2019. La cacciatrice di fossili. Mary Anning si racconta. Trieste: Editoriale Scienza.

Tarantini, Massimo 2008. "Il neolitico. La prima grande trasformazione." Mundus. Rivista di didattica della storia I (I): 74-165. 
